\title{
Spinal Cord Ischemia After Endovascular Aortic Repair - A Case Report
}

\section{Raquel Reis Soares $^{1 *}$ | Leonardo Ferber Drumond ${ }^{2}$ | Giovana Freitas Rocha de Souza ${ }^{3}$ | Lucas Valbon de Azeredo Coutinho4 ${ }^{4}$ Joao Victor Tavares de Mendonça Garretto ${ }^{4}$ | Orlando Pinto Gontijo Neto $^{3}$ | Daniel Soares da Mata $^{5}$ | Luiza Miraglia Firpe ${ }^{5}$ Matheus Ferber Drumond ${ }^{2}$}

*Correspondence: Raquel Reis Soares

Address: ${ }^{1}$ Cardiovascular Anesthesiologist of Biocor Institute, Brazil; ${ }^{2}$ Cardiovascular Surgeon of Biocor Institute, Brazil; ${ }^{3}$ Cardiovascular Anesthesiology Resident, Brazil; ${ }^{4}$ Cardiovascular Surgery Resident, Brazil; ${ }^{5}$ Medicine Student at Faculdade de Medicina de Barbacena, Brazil

e-mail $\bowtie$ : raquelr.soares@hotmail.com

Received: 25 June 2021; Accepted: 02 July 2021

Copyright: (C) 2021 Raquel RS. This is an open-access article distributed under the terms of the Creative Commons Attribution License, which permits unrestricted use, distribution, and reproduction in any medium, provided that the original work is properly cited.

\begin{abstract}
Spinal cord ischemia (SCI) is a devastating complication after aortic aneurysm repair. Even in a less invasive procedure, as endovascular aneurysm treatment, paraplegia is still a concern. SCI is associated with perioperative hemodynamic instability and damage of the main or collateral spinal cord circulation. This is a case report of a patient with complicated aortic dissection who underwent several procedures (open surgeries and endovascular treatments) and developed paraplegia after the last endovascular repair. We highlight the cerebrospinal fluid drainage as a therapeutic procedure rather than a prophylactic one, associated with maintenance of hematimetric levels, permissive hypertension and administration of L-type calcium inhibitor. We also discuss spinal cord perfusion and motor evoked potentials monitoring during aortic aneurysm repair. The patient's condition improved, he was discharged home 15 days after the procedure. Three months after discharge the patient was able to walk alone without difficult.

Keywords: Thoracoabdominal Aortic Aneurysm, Endovascular Aneurysm Repair, Paraplegia, Spinal Cord Ischemia, Cerebrospinal Fluid Drainage
\end{abstract}

\section{Introduction}

The thoracoabdominal aortic aneurysms (TAAAs) repair exposes the spinal cord to ischemic injuries (Svensson et al., 1993), which varies between 3.9\% and 31\% in the published literature (Maurel et al., 2015; Dias et al., 2015). The endovascular repair of TAAAs with fenestrated and branched stent grafts has increased acceptance among vascular surgeons and gradually became the first-line treatment in some centers with a great expectation to provide a survival benefit and also prevent spinal cord ischemia (SCI) (Verhoeven et al., 2015; Ferreira et al., 2008). Although the minimally invasive character of the procedure, spinal cord injury still is a catastrophic complication.

As spinal cord blood flow (SCBF) depends on the difference between mean arterial pressure (MAP) 
and the intrathecal pressure (ITP), cerebrospinal fluid drainage CSFD (keeping liquoric pressure below $10 \mathrm{mmHg}$ ) is largely utilized prophylactically in open repair surgery for TAAA to avert SCI (improving spinal cord perfusion).

During endovascular procedures, however, its role is not clear. The preoperative placement of CSFD is associated with many neurologic complications and unsatisfactory outcomes concerning neurologic aspects, such as spinal hematomas, intracranial hemorrhage, intra-cranial hypotension, bloody drainage, drain fracture, CSF leakage, severe headache (Oderich and Tenorio, 2020). As the paraplegia following endovascular procedures is most commonly observed in the late post-operative period (Greenberg et al., 2008), it is possible to place the CSFD lately, just in case the patient presents neurologic symptoms.

We present the case of a patient who underwent endovascular repair of aortic dissection progression and developed paraplegia on the immediate postoperative period. CSFD was placed for treatment of paraplegia. The patient's progress was encouraging. He recovered from the paraplegia and was discharged from the hospital 15 days later with an improved neurological status.

\section{Case Report}

A 66 year-old-man with a history of arterial hypertension, dyslipidemia, chronic obstructive pulmonary disease had previously undergone a Bentall de Bono plus aortic arch treatment plus elephant trunk procedure for correction of acute type A aortic dissection in 2010. In 2015 a huge enlargement of the false lumen of the descending aorta (diameter of $75 \mathrm{~mm}$ ) was treated by an endoprosthesis since the elephant trunk implanted in 2010 till $25 \mathrm{~cm}$ from the celiac trunk, to preserve radicular magna artery area (Fig. 1). In September 2020 he presented dysphagia and weight loss. After gastrointestinal propaedeutic, a false lumen enlargement (140 mm) compressing the distal esophagus (Fig. 2) was discovered. In January 2021 a second TEVAR procedure was done, using two endoprosthesis, one a covered endoprosthesis and the other petit coat concept endoprosthesis.

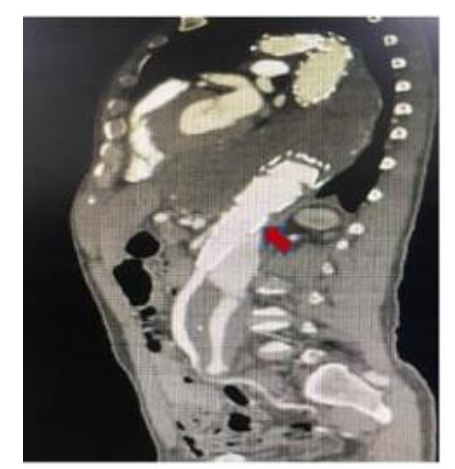

Figure 1: Lateral Incidence of CT angiography. Presence of elephant trunk in aortic arch endoprosthesis in thoracic aorta. False light with important flow (Arrow) 


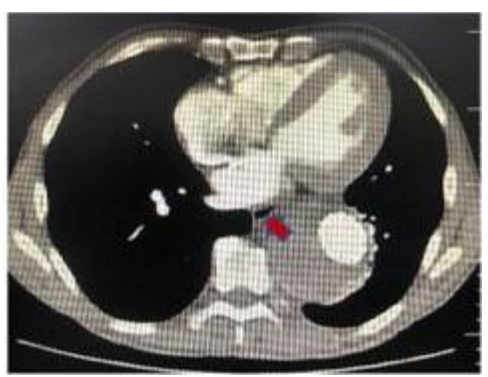

Figure 2: Compression of intrathoracic structures, such as the distal esophagus (Arrow)

In March 2021 the patient presented sudden back pain and paraplegia spontaneously reversed in two hours. He was sent to the intensive care unit (ICU) anticoagulated and maintained with permissive hypertension (mean arterial pressure - MAP>100 mmHg). The angio-tomography showed important compression of the iliac arteries by the false lumen dissection. The right renal artery emerged totally from the false lumen (Fig. 3).

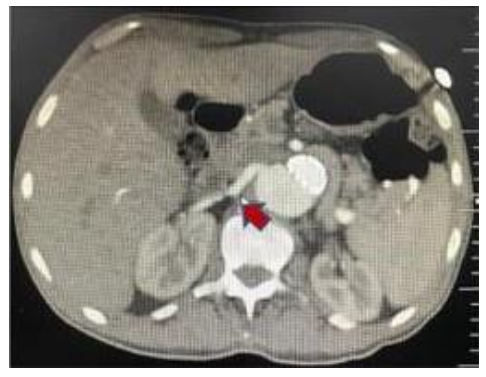

Figure 3: Right renal artery emerging by false aneurysm light

After assignment of informed consent and discussion with the family about risks, including paraplegia and the necessity of the placement of a CSFD, we decided for a new endovascular procedure. In the perioperative period, under general anesthesia, MAP remained above $85 \mathrm{mmHg}$, hematocrit above $30 \%$ and methylprednisolone $(30 \mathrm{mg} / \mathrm{kg}$ ) was administered to prevent the Postimplantation Syndrome. An infra-renal endoprosthesis treated the iliac vessels, maintaining total perfusion of both, external and internal iliac arteries. A recovered stent treated the right renal artery, recommunicating it with the true aortic lumen. The flow in the aortic segment between the celiac trunk and the renal arteries remained through the false and the true lumens (Fig. 4).

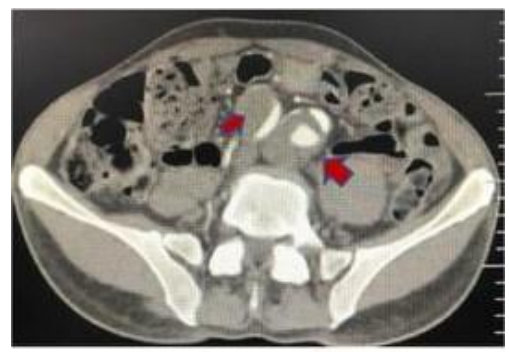

Figure 4: Lliac arteries after their division. Major theft of flow by dissector light (Arrows) 
The patient was extubated immediately after the procedure but woke up paraplegic. Prompt examination by an experienced neurologist found complete paralysis of the legs, total loss of pinprick and vibratory sensation. As the patient had all the lower limb pulses present and normal, thrombosis diagnosis was discarded. A clinical diagnosis of SCI was made. A CSFD catheter was immediately inserted, maintaining liquoric pressure < $10 \mathrm{~cm} \mathrm{H20}$, MAP above $90 \mathrm{~mm} \mathrm{Hg}$, and oxygen transport optimized (hematocrit above 30\% and hemoglobin oxygen saturation above 90\%), a L-type calcium inhibitor was prescribed. An improvement of neurologic function occurred in the first hours. The catheter was removed 72 hours later. The mean drainage volume was $120 \mathrm{ml} / 24 \mathrm{hs}$. The patient received twice-daily physiotherapy treatment for rehabilitation and was discharged from the hospital 15 days after the procedure. One month after the discharge he was beginning to walk again. Three months after discharge the patient was able to walk alone without difficult.

\section{Discussion}

Elective endovascular repair is associated with lower perioperative morbidity and mortality than open repair but SCI also remains a devastating complications (Dijkstra et al., 2018; Huang et al., 2018). Neurological deficits, such as urinary and/or fecal incontinence, paraparesis and paraplegia occur in 5.7\% of cases (Dijkstra et al., 2018; Huang et al., 2018). Furthermore, affected patients have increased lethality rates and decreased quality of life (Wortmann et al., 2017). The SCI incidence in open surgery can reach $20 \%$, while in endovascular therapy it varies between $1.2 \%$ to $14 \%$ and in patients with a Crawford II aneurysm, about 16\% (Wortmann et al., 2017; Keith Jr et al., 2012).

Paraplegia is explained by medullary ischemia, either by arterial hypotension, mainly or collateral irrigation occlusion or thromboembolism (Hiratzka et al., 2010). The percentage of thoracic aortic coverage was the most powerful determinant of SCI (Wortmann et al., 2017; Bisdas et al., 2015). 10,13 Other risk factors were: the aortic segment to be treated (major risk between T9-T12 due to the emergence of great anterior radicular artery) (Wortmann et al., 2017; Keith Jr et al., 2012), chronic kidney disease, perioperative arterial hypotension, female gender, surgery duration (Wortmann et al., 2017).

The techniques for spinal cord protection improves spinal perfusion and includes: permissive arterial hypertension (MAP> $90 \mathrm{mmHg}$ in the perioperative period), reduce surgical duration, staged procedures (it is expected the development of collateral vessels for spinal irrigation), aneurysm sac irrigation, optimization of hematimetric levels (hemoglobin above $10 \mathrm{mg} / \mathrm{dL}$ ) and oxygen saturation in arterial blood (above 90\%), electrophysiological monitoring, permissive hypothermia, perioperative CSF drainage and drug therapy (L-type calcium inhibitors, intrathecal papaverine, corticosteroids, mannitol, 
naloxone) (Wortmann et al., 2017; Keith Jr et al., 2012; Hiratzka et al., 2010).

Spinal cord injury is a complex pathological process. Several mechanisms contribute to the injury release of excitatory amino acids, intracellular accumulation of calcium, apoptos is of neurons, generation of intracellular free radical, disruption of membrane structure and function, increased expression of ICAM-1, IL-1, IL-8, adhesion and recruitment of leukocytes, inflammation (Xiong et al., 2011).

The spinal cord perfusion has a very complex and variable arterial supply in each patient. Besides the great radicular artery, there are the anterior spinal artery (originated from the vertebral arteries), two posterior spinal arteries, of the same origin, and segmental branches. Collateral irrigation includes: intercostal branches, lumbar arteries and plexus formed by epidural arteries and those that irrigate the paravertebral muscles. All vessels are interconnected and have important anastomoses with arteries originating from the subclavian arteries, cranially, and hypogastric arteries in the caudal direction (Amato and Stolf, 2015). According to Griepp, et al., if the hypogastric or subclavian arteries flow is interrupted during a thoracoabdominal aneurysm surgery, a greater risk of ischemia is present (Griepp et al., 2012). The concept of the importance of the spinal cord's collateral blood supply network and its imbalance is the key to understand the SCI after endovascular repair of TAAAs.

It is worth remembering that the decompression process performed by CSF drainage decreases intrathecal pressure, and facilitates blood supply to a lower pressure zone.

Electrophysiology is useful to perioperative management of patients considered to be at high risk for ischemic spinal involvement, and to minimize the risk of ischemia (Sloan et al., 2013). Cell's death occurs within 3-4 hours when the perfusion pressure is below the ischemic threshold and the metabolism is normal (Sloan et al., 2013). Without perfusion, cell's death occurs after 3 to 5 minutes (Sloan et al., 2013). This monitoring can be an early warning of flow at critical levels (Sloan et al., 2007). Somatosensory evoked potential (SSEP) is used to monitor spinal activity (Sloan and Jameson, 2007). SSEP are produced in response to electrical stimulation of peripheral motor and sensitive nerves in the wrist and ankle, following the path of proprioception and vibratory sensitivity (Sloan et al., 2013; Sloan et al., 2007).

The second electrophysiological monitoring technique would be the motor evoked potential (MEP), which is more sensitive to medullary ischemia in the territory of the anterior spinal artery (which supplies the motor tracts) than SSEP (Griepp et al., 2012). The neural pathway originates in pyramidal cells of the motor cortex and descends through the cortico-spinal tract until it makes synapses in the cells of the anterior horn of the spinal cord (Sloan et al., 2007). However, MEP is more vulnerable to the action 
of halogenates (up to 0.5 MAC may be required) and neuromuscular blockers (Sloan et al., 2013). Clinical studies prove that when comparing SSEP and PEM, there is a relatively long delay (7 to 30 minutes) between the onset of ischemia and the disappearance of the SSEP response, while the PEM changes after about 2 to 5 minutes (Sloan et al., 2007).

There is no consensus regarding the use of perioperative CSFD in endovascular aortic surgery, and the following scenarios can be considered: 1) preoperative approach with catheter implantation in all patients 2) preoperative management with catheter implantation in patients considered to be at high risk for spinal ischemia 3) implantation of a catheter in the postoperative period in patients who developed spinal ischemia (Wortmann et al., 2017; Keith Jr et al., 2012).

Since Coselli's study in 2002, CSFD has become the protocol for several treatment centers for aortic diseases (Coselli et al., 2002). CSFD has been used to decrease the intra-spinal canal pressure, thereby increasing spinal cord perfusion pressure (Weissler et al., 2020). However, the effectiveness is controversial and there is a lack of data in the literature (Khan and Stansby, 2012). More recent studies question the prophylactic use of CSF drainage in endovascular surgery, since it's associated with significant complications, such as presence of hemorrhagic CSF , CSF fistula, drainage catheter fracture, post-dural puncture headache, subarachnoid hemorrhage, epidural hematoma, meningitis, neurological deficit, and even death by intracranial hypotension or intracranial hemorrhage (Oderich and Tenorio, 2020). A recent study from the Mayo Clinic (Kärkkäinen et al., 2020) found a 9\% rate of CSFD-related complications, including severe, potentially life-threatening complications in $4 \%$, and one-third of the spinal cord ischemia in their study were actually caused by CSFD. Surgery cancellation by hemorrhagic puncture, and prolongation of the length stay in Intensive Care Unit are also relevant factors when analyzing the risk / benefit of the procedure (Kärkkäinen et al., 2020).

Compared with open procedures, delayed paralysis/paresis is the predominant presentation of SCI in endovascular aneurysm repair. Considering the minor aggressive character of the procedure, this fact would be expected. The later deficit challenges the efficacy of perioperative electrophysiological monitoring to prevent spinal cord ischemia in the endovascular treatment of thoracoabdominal aneurysm (Bisdas et al., 2015).

Some studies demonstrate that the use of CSFD as a therapeutic technique in the context of postoperative paraplegia would have a better result than its preoperative prophylactic use $(1.5 \%$ risk of permanent paraplegia in postoperative therapeutic drainage versus $2,5 \%$ of preoperative prophylactic) (Hiratzka et al., 2010). The performance of CSFD in the postoperative period is usually possible, safe and improves the patient's clinical condition, in addition to avoiding unnecessary drainage in $80-90 \%$ of 
patients who would have an evolution without complications (Oderich and Tenorio, 2020).

Based on the data currently known, we chose to perform the procedure on our patient without preoperative CSFD. Emergent CSFD placement is available 24/7 in our institution due to neurosurgery and anesthesiology duty. The patient was promptly evaluated by the neurology team upon awakening from anesthesia with paraplegia. The placement of CSFD was carried out immediately and maintained for $72 \mathrm{~h}$. The patient's neurological condition improved and he was discharged home 15 days after the procedure. Three months after discharge the patient was able to walk alone without difficult.

It is important to emphasize that, in view of the seriousness of the cases of complex and evolutionary aortic pathologies, the precise diagnosis, the understanding of the pathophysiology of spinal cord perfusion, the planning as well as the careful conduct and the integrated efforts of the specialties involved, aiming to improve the patient's evolution and prognosis.

Endovascular aneurysm repair is also associated to a systemic inflammatory response in a significant number of cases, the Postimplantation Syndrome, the patient presents fatigue, fever and laboratory findings of inflammation, prevention may be achieved with perioperative administration of 30 $\mathrm{mg} / \mathrm{kg}$ of methylprednisolone (De La Motte et al., 2014).

\section{Conclusion}

Aortic diseases are complex pathologies with high surgical morbi-mortality rates. The endovascular surgical repair reduced these rates, but the most devastating complication, spinal cord ischemia, continues to be a source of concern.

The importance of CSFD for prevention of SCI after thoracic endovascular aortic repair (TEVAR) remains unclear (Hiratzka et al., 2010). Permissive hypertension and collateral revascularization may allow avoidance of CSFD and its risks (Weissler et al., 2020). Since the spinal cord has multiple vascular collaterals, the probability of SCI arising from an interruption of a certain blood supply is low, unless obstruction to blood supply occurred on multiple levels. A systematic review shows a drainage-related mortality of nearly $1 \%$ and a pooled event rate of $6.5 \%$ for others complications (Rong et al., 2018). It suggests that the prophylactic CSFD may cause as many problems as it can avert (Weissler et al., 2020).

In this case we presented, a progression in aortic dissection treated with endovascular approach, the patient's left subclavian artery was open and we used permissive arterial hypertension in order to improve spinal perfusion. The immediate postoperative paraplegia was treated by CSFD, permissive arterial hypertension, optimization of oxygen transport and the use of a L-type calcium inhibitor. The 
patient recovered from the paraplegia and was followed by the neurologic and physiotherapy staff.

We believe that the early intervention was the key of the success and that the spinal cord perfusion comprehension in endovascular aneurysm repair is a challenge for the medical team. The use of CSFD as a therapeutic option, rather than a prophylactic practice, was supported by the favorable outcome of this clinical case. The use of intraoperative monitoring of motor evoked potentials would detect earlier ischemic episodes in order to prevent paraplegia. The evoked potential monitoring is part of the endovascular TAAA repair protocol in reference centers (Weissler et al., 2020).

\section{References}

Amato ACM and Stolf NAG. Anatomy of spinal blood supply. J Vasc Bras 2015; 14: 248-252.

Bisdas T, Panuccio G, Sugimoto M, Torsello G, Austermann M. Risk factors for spinal cord ischemia after endovascular repair of thoracoabdominal aortic aneurysms. J Vasc Surg 2015; 61: 1408-1416.

Coselli JS, LeMaire SA, Köksoy C, Schmittling ZC, Curling PE. Cerebrospinal fluid drainage reduces paraplegia after thoracoabdominal aortic aneurysm repair: results of a randomized clinical trial. J Vasc Surg 2002; 35: 631-639.

De La Motte, Kehlet H, Vogt K, Nielsen CH, Groenvall JB, Nielsen HB, et al. Preoperative methylprednisolone enhances recovery after endovascular repair: A randomized, double-blind, placebo-controlled clinical trial. Annals of Surg 2014; 260: 540549.

Dias NV, Sonesson B, Kristmundsson T, Holm H, Resch T. Short-term outcome of spinal cord ischemia after endovascular repair ofthoracoabdominal aortic aneurysms. Eur J Vasc Endovasc Surg 2015; 49: 403-409.

Dijkstra ML, Vainas T, Zeebregts CJ, Hooft L, van der Laan MJ. Editor's Choice - Spinal Cord Ischaemia in Endovascular Thoracic and Thoraco-abdominal Aortic Repair: Review of Preventive Strategies. Eur J Vasc Endovasc Surg 2018; 55: 829-841.

Ferreira M, Lanziotti L, Monteiro M. Branched devices for thor-acoabdominal aneurysm repair: early experience. J Vasc Surg 2008; 48: 30-36.

Greenberg RK, Lu Q, Roselli EE, Svensson LG, Moon MC, Hernandez AV et al. Contemporary analysis of descending thoracic and thoracoabdominal aneurysm repair. Circulation 2008; 118: 808-817.

Griepp EB, Di Luozzo G, Schray D, Stefanovic A, Geisbüsch S, Griepp RB. The anatomy of the spinal cord collateral circulation. Ann Cardiothorac Surg 2012; 1: 350-357.

Hiratzka LF, Bakris GL, Beckman JA, et al. ACCF/AHA/ AATS/ACR/ASA/SCA/SCAI/SIR/STS/SVM guidelines for the diagnosis and management of patients with thoracic aortic disease: executive summary. A report of the American College of Cardiology Foundation/American Heart Association Task Force on Practice Guidelines, American Association for Thoracic Surgery, American College of Radiology, American Stroke Association, Society of Cardiovascular Anesthesiologists, Society for Cardiovascular Angiography and Interventions, Society of Interventional Radiology, Society of Thoracic Surgeons, and Society for Vascular Medicine. Catheter Cardiovasc Interv 2010; 76: E43e86.

Huang Q, Chen XM, Yang H, Lin QN, Qin X. Effect of Left Subclavian Artery Revascularisation in Thoracic Endovascular Aortic Repair: A Systematic Review and Meta-analysis. Eur J Vasc Endovasc Surg 2018; 56: 644-651.

Kärkkäinen JM, Cirillo-Penn NC, Sen I, Tenorio ER, Mauermann WJ, Gilkey GD, Kaufmann TJ, Oderich GS. Cerebrospinal fluid drainage complications during first stage and completion fenestrated-branched endovascular aortic repair. J Vasc Surg 
2020; 71: 1109-1118.

Keith Jr CJ, Passman MA, Carignan MJ, Parmar GM, Nagre SB, Patterson MA, Taylor SM, Jordan Jr WD. Protocol implementation of selective postoperative lumbar spinal drainage after thoracic aortic endograft. J Vasc Surg 2012; 55 :1-8.

Khan SN and Stansby G. Cerebrospinal fluid drainage for thoracic and thoracoabdominal aortic aneurysm surgery. Cochrane Database Syst Rev 2012; 10: CD003635.

Maurel B, Delclaux N, Sobocinski J, Hertault A, Martin-Gonzalez T, Moussa M, Spear R, Le Roux M, Azzaoui R, Tyrrell M, Haulon S. Editor's choiced. The impact of early pelvic and lowerlimb reperfusion and attentive peri-operative management on theincidence of spinal cord ischemia during thoracoabdominal aorticaneurysm endovascular repair. Eur J Vasc Endovasc Surg $2015 ; 49: 248-254$.

Oderich GS and Tenorio ER. To drain or not to drain? That is the question. Vascular Specialist;15th June 2020

Rong LQ, Kamel MK, Rahouma MK, White RS, Lichtman AD. Cerebrospinal-fluid drain-related complications in patients undergoing open and endovascular repairs of thoracic and thoraco-abdominal aortic pathologies: a systematic review and metaanalysis. British Journal of Anaesthesia 2018; 120: 904e913.

Sloan TB and Jameson LC. Electrophysiologic Monitoring During Surgery to Repair the Thoraco-Abdominal Aorta. J Clin Neurophysiol 2007; 24: 316-327.

Sloan TB, Edmonds HL, Koht A. Intraoperative Electrophysiologic Monitoring in Aortic Surgery. J Cardiothorac Vasc Anesth 2013; $27: 1364-1373$.

Svensson LG, Crawford ES, Hess KR, Coselli JS, Saf iHJ. Experience with 1509 patients undergoing thoracoabdominal aorticoperations. J Vasc Surg1993;17(2):357e68.

Verhoeven EL, Katsargyris A, Bekkema F, Oikonomou K, Zeebregts CJ, Ritter W, et al. Ten-year experience with endovascularrepair of thoracoabdominal aortic aneurysms: results from 166consecutive patients. Eur J Vasc Endovasc Surg 2015; 49: 524-531.

Weissler EH, Voigt SL, Raman V, Jawitz O, Doberne J, Anand J, Plichta R, Gaca JG, McCann RL, Hughes GC. Permissive Hypertension and Collateral Revascularization May Allow Avoidance of Cerebrospinal Fluid Drainage in Thoracic Endovascular Aortic Repair. Ann Thorac Surg 2020; 110: 1469-75.

Wortmann M, Böckler D, Geisbüsch P. Perioperative cerebrospinal fluid drainage for the prevention of spinal ischemia after endovascular aortic repair. Gefässchirurgie 2017; 22: S35-S40.

Xiong M, Chen S, Yu H, Liu Z, Zeng Y, Li F. Neuroprotection of erythropoietin and methylprednisolone against spinal cord ischemia-reperfusion injury. J Huazhong Univ Sci Technolog Med Sci 2011; 31: 652. 